TITLE:

\title{
An attempt to correct strain data measured with vault-housed extensometers under variations in temperature
}

\author{
$\operatorname{AUTHOR}(\mathrm{S})$ :
}

Yamazaki, Ken'ichi

\section{CITATION:}

Yamazaki, Ken'ichi. An attempt to correct strain data measured with vault-housed extenso meters under variations in temperature. Tectonophysics 2013, 599: $89-96$

ISSUE DATE:

2013-06

URL:

http://hdl.handle.net/2433/174325

\section{RIGHT:}

(c) 2013 Elsevier B.V.; この論文は出版社版でありません。引用の際には 出版社版をご確認ご利用ください。; This is not the published version. Please cite only the published version. 


\title{
An attempt to correct strain data measured with vault-housed extensometers under variations in temperature
}

\author{
Ken'ichi Yamazaki ${ }^{\mathrm{a}, *}$ \\ ${ }^{a}$ Miyazaki Observatory, Research Center for Earthquake Prediction, Disaster Prevention Research Institute, Kyoto University, 3884 Kaeda, \\ Miyazaki 889-2161, Japan
}

\begin{abstract}
Strain data obtained by vault-housed extensometers have precisions on the order of nanostrains, but they are distorted by variations in temperature, which cause two types of noise: "actual variations" due to the thermo-elastic effect of the Earth's crust, and "false variations" due to the thermal expansion of extensometer, which occurs when the extensometers themselves are subjected to variations in temperature. Here, I explore a method of removing false variations, which are severe when the vault is located at shallow depths. If variations in temperature at arbitrary points inside a vault are estimated, false variations can be removed from the recorded variations in strain. I derive formulae that enable variations in temperature to be estimated at various points in a vault, based on measured variations at reference points. The formulation is valid if some simplification is allowed. I examined whether variations in temperature inside a vault can be estimated in terms of the derived formulae, and obtained the following results. When the reference temperature data are obtained from adequate points in the vault, variations in temperature at another point can be estimated with an accuracy of $0.1^{\circ} \mathrm{C}$. However, when the reference temperature data are obtained from outside the vault, estimated variations in temperature are rather inaccurate, which means that the false variations in strain cannot be removed accurately. Moreover, the data indicate that the thermal diffusivity of the ground is temporally variable, and this introduces another difficulty in correcting false variations in strain data. These results indicate that correcting the distortions in strain data due to variations in temperature is much more difficult than anticipated.
\end{abstract}

Keywords: Data correction, Vault-housed extensometer, False variations in strain, Thermal expansion, Heat conduction, Reference temperatures

\section{Introduction}

Following on from studies of crustal deformation in the 1960s (e.g., Hagiwara and Rikitake, 1967), observations of crustal deformation using extensometers (i.e. rods with a length of several tens of meters) housed in vaults (i.e. long tunnels) have been considered to be one of the important ways of measuring deformation of the Earth's crust. The advantage of extensometers in vaults over other modern geodetic techniques, such as Global Positioning Systems (GPS), Interferometric Synthetic Aperture Radar (InSAR), and bore-hole type strain meters and tiltmeters, is their ability to detect

*Miyazaki Observatory, Research Center for Earthquake Prediction, Disaster Prevention Research Institute, Kyoto University, 3884, Kaeda, Miyazaki 889-2161, Japan

Email address: kenichi@rcep.dpri.kyoto-u.ac.jp (Ken'ichi Yamazaki)

Preprint submitted to Tectonophysics small signals. This is particularly the case for detecting events in the period range of seconds to hours. Extensometers in vaults installed under excellent conditions can detect subtle changes in strain of the order of $10^{-9}$, such as those caused by the Earth's free oscillation (e.g., Park et al., 2008), the pre-eruption processes of volcanoes (e.g., Ishihara, 1990; Yamazaki et al., 2012), and the steps in strain due to remote earthquakes (Yamazaki et al., 2011). These subtle changes are barely detectable by other means. Crustal strain data measured with vault-housed extensometers can also be used for studying events in the pre-GPS era, because of their rather long history. For these reasons, attempts to reexamine crustal strain data have been started, and data exchange systems are under construction (Yamaguchi et al., 2010).

However, strain data obtained with vault-housed extensometers have several drawbacks when they are used for studies of long-term (i.e., $\&$ days) events. One of the

June 5, 2013 
major drawbacks is the distortion of data due to temporal variations in temperature, which give rise to two types of noise in strain data measured by extensometers: actual deformation of the crust caused by changes in temperature (e.g. Berger, 1975; Harrison and Herbst, 1977) and false variations in strain caused by the thermal expansion of sensor rods (e.g., Furuzawa et al., 1993). Hereafter, I refer to these noises as the "thermoelastic effect" and "false variation in strain (due to variations in temperature)", respectively. Strategies to avoid the thermo-elastic effect have been proposed (e.g., BenZion and Leary, 1986), although this task is generally difficult because the effect represents the integrated effect of thermal expansion of the ground, and is therefore inevitable even if we refer to temperature data at a point.

False variations in strain due to variations in temperature arise by the following mechanism. Extensometers are composed of a rod, one end of which is fixed and the other (the free end) unfixed (Fig. 1). Sensors of extensometers measure the displacement of the ground relative to the free-end of the rod. Although the sensor records the ground strain, it also records changes in sensor length. The length of a rod changes with temperature; thus, false changes in strain may be recorded. In contrast to the thermo-elastic effect, false variations in strain have not been investigated in detail because they are easily avoided by installing the sensor at great depth in a long tunnel, where temperature variations are negligible. Nevertheless, some extensometers used for geodetic purposes are installed in shallow vaults (e.g. Teraishi et al., 2009).

Establishing a method for the reliable correction of strain data is indispensable if data from shallow vaults are to be used. In general, the main drawbacks in using extensometer strain data are considered not to be the false variation in strain due to variations in temperature, but to be the loadings that result from precipitation and groundwater (e.g., Kasahara et al., 1983). Accurate corrections for the effects of ground water are obviously difficult because the relevant relationships are highly non-linear (e.g., Hashimoto, 2007). The thermo-elastic effect is also difficult to remove, as mentioned above. Compared with these complex effects, one might anticipate that corrections for false variations in strain due to variations in temperature would be relatively easy to make because the spatio-temporal variations in temperature can be described by a linear equation. If such corrections for temperature are possible, we can choose the option of a shallow vault for an extensometer because a shallow vault, even though strongly affected by temperature variations, is relatively unaffected by groundwater. However, few studies have attempted to quantify the ef- (a)

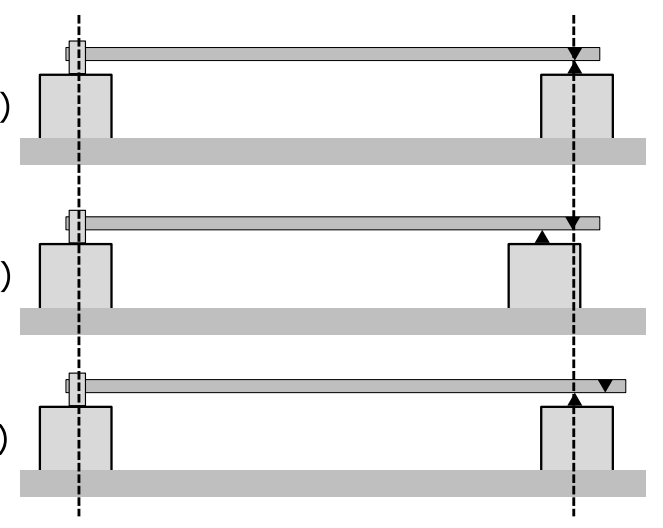

Figure 1: Schematic of an extensometer, showing the mechanism by which false variations in strain are generated due to variations in temperature. (a) Original location of free end of the extensometer and sensor mounted on the ground. (b) The case where the ground contracts because of tectonic phenomena. (c) The case where the ground does not contract, but the rod of the extensometer is extended due to thermal expansion. The sensor only records the relative displacements between the two triangles in each panel, and it therefore cannot distinguish (b) from (c).

fects of temperature variations on extensometers, or to clarify the validity of the data corrections used.

The purpose of this paper is to determine whether data corrections can be reliably made for strain data obtained from vault-housed extensometers where there are significant variations in temperature. Although the thermo-elastic effect is generally dominant as a source of distorted strain data due to variations in temperature, false variations in strain due to variations in temperature are considered to be larger in the case of extremely shallow vaults. For this reason, the present study focuses on false variations in strain; the thermo-elastic effect is only briefly discussed. To this end, the remainder of this paper is organized as follows. In Section 2, we consider theoretically how and when we can remove the false variations in strain due to variations in temperature. In Section 3, we present a set of temperature and strain data used for numerical testing, obtained at a tectonically active location but which were probably distorted by temperature variations. In Section 4, the procedure of estimating variations in temperature inside vaults is applied to correct strain data, and determine whether the procedure works successfully. In Section 5 , we discuss the usefulness of the strain data measured in shallow vaults, based on the results presented in Section 4. Finally, the main conclusions are summarized in Section 6. 


\section{Strategies and the actual procedure of data cor- rection}

Given that false variations in strain data $(\Delta E)$ arise from variations in temperature $(\Delta T)$, a necessary condition for $\Delta E$ being removable is that $\Delta T$ can be estimated by some reference time series. Therefore, we should consider the problem of estimating $\Delta T$ at one location $(\boldsymbol{x}$; referred to as the target point) by using $\Delta T$ at another location ( $\boldsymbol{x}_{\mathrm{ref}}$; referred to as the reference point). If $\Delta T(x, t)$, where $t$ denotes time, can be estimated by $\Delta T\left(\boldsymbol{x}_{\mathrm{ref}}, t\right)$, the following relationship should be satisfied:

$$
\Delta T(\boldsymbol{x}, t)=\int_{0}^{\infty} A\left(\boldsymbol{x}, \boldsymbol{x}_{\mathrm{ref}} ; s\right) \Delta T\left(\boldsymbol{x}_{\mathrm{ref}}, t-s\right) d s,
$$

either in a time domain, or equivalently,

$$
\Delta T(\boldsymbol{x}, \omega)=A\left(\boldsymbol{x}, \boldsymbol{x}_{\mathrm{ref}} ; \omega\right) \Delta T\left(\boldsymbol{x}_{\mathrm{ref}}, \omega\right)
$$

in a Fourier domain. The function $A$ describes a predictive filter of temperature.

To estimate $\Delta T$ at an arbitrary point $\boldsymbol{x}$, it is necessary to find the optimum form of $A$ for a given set of $\boldsymbol{x}$ and $\boldsymbol{x}_{\text {ref }}$. A straightforward procedure to determine $A$ is an empirical method using Eqs (1) or (2). Using actual data of temperature at $\boldsymbol{x}$ and $\boldsymbol{x}_{\text {ref }}$, the filter function $A$ in Eq. (1) or (2) can be determined in principle. However, in practice, it is difficult to determine $A$ correctly by empirical methods. Temporal variations in temperature generally involve a large range of frequency (i.e., daily to decadal), and this means that $A$ cannot be determined unless $T$ data are available for a period of several decades. Given that $T$ is usually available only for a restricted period of time, the empirical determination becomes difficult.

Accurate determination of $A$ in Eq. (1) is feasible only if $A$ is expressed by a known function characterized by a small number of parameters. In such a case, we need to determine only the relevant parameters. The existence of such a function is uncertain, but if it exists, the explicit form of the function should be determined by a theoretical consideration of heat conduction in the ground surrounding the vault under observation. If such a function does not exist, or if the function involves a large number of parameters, determination of $A$ becomes difficult.

Heat conduction in the ground is expressed as

$$
\frac{\partial}{\partial t} T(\boldsymbol{x}, t)=k(\boldsymbol{x}, t) \nabla^{2} T(\boldsymbol{x}, t),
$$

where $k$ is thermal diffusivity. We need to express the solution of Eq. (3) in a form of Eq. (1). Since $k$ has three-dimensional heterogeneity, and because the explicit distribution of $k$ is difficult to estimate, finding a solution for $T$ is generally difficult. An exceptional case is that where the heat conduction equation is reduced to the form

$$
\frac{\partial}{\partial t} T\left(x^{\prime}, t\right)=k\left(x^{\prime}\right) \frac{\partial^{2}}{\partial x^{\prime 2}},
$$

where $x^{\prime}$ is a scalar function of $\boldsymbol{x}$. This simplification applies to situations where $\mathrm{k}$ has a one-dimensional (1D) distribution. For this reason, herein we refer to the simplification in Eq. (4) as a 1-D approximation. Note that Eq. (4) may be valid only within a restricted region of the ground (e.g., Fig. 2).

If this reduction is possible, the equation is further reduced to

$$
\frac{\partial}{\partial t} T(x, t)=\frac{\partial^{2}}{\partial x^{2}} T(x, t),
$$

by putting a new variable $x$ as $x=k\left(x^{\prime}\right)^{-1 / 2} x^{\prime}$. Since Eq. (5) does not include thermal diffusivity, we can ignore the spatial distribution of $k$. In this case, we can refer to the literature for existing solutions of heat conduction in a solid with a uniform thermal diffusivity. Below, we enumerate some explicit solutions of Eq. (5) for certain situations. The listed formulae are derived with the use of equations in Carslaw and Jaeger (1986).

First, consider the case where reference data inside the vault $(x$ sre $f>0)$ are available. If the heat conduction between $x$ and $x \operatorname{sre} f\left(x=x_{\text {ref }}+\Delta x\right)$ is described by Eq. (2), the function $A$ in Eq. (1) is given by

$$
A\left(x, x_{\text {ref }} ; \omega\right)=\exp [-p(\omega) \Delta x]
$$

in a frequency domain, where

$$
p(\omega)=-\sqrt{\frac{\omega}{2}}(1+i),
$$

and

$$
A\left(x, x_{\text {ref }} ; t\right)=\frac{\Delta x}{\sqrt{4 \pi t^{3}}} \exp \left(-\frac{\Delta x^{2}}{4 t}\right)
$$

in a time domain (see, for example, section 2.5 of Carslaw and Jaeger, 1986).

Note that Eq. (1) with $A$ given by Eq. (8) is valid only when $\Delta x>0$ (i.e., $x>x_{\text {ref }}$ ). Therefore, we can estimate $T(x, t)$ only when reference data $T\left(x_{\mathrm{ref}}, t\right)$ for $x>x_{\text {ref }}$ are available.

Next, we consider the case where reference temperatures are only available outside the vault (i.e., $x$ sref $=$ $0)$. In this case, we must explicitly consider the boundary condition at the ground surface (i.e., $x=0$ ). To balance the heat flow at this level, the following condition should be satisfied:

$$
\lambda\left[T_{\text {ext }}(t)-T(x=0, t)\right]+Q(t)=\frac{\partial}{\partial x} T(x=0, t),
$$


where $\lambda$ is the thermal conductivity of the ground and $Q$ represents heat generation at the ground surface. The heat generation $Q$ is difficult to determine because it depends on the intensities of solar radiation and the absorption coefficients of the ground, both of which are generally unknown. Therefore, the external temperature ( $\left.T_{\text {ext }}\right)$ is only used for reference if $Q$ can be ignored. Below, we assume the situation $Q=0$, so that

$$
\lambda\left[T_{\mathrm{ext}}(t)-T(x=0, t)\right]=\frac{\partial}{\partial x} T(x=0, t) .
$$

In this case, the function $A$ in Eqs (1) and (2) is described by

$$
A\left(x, x_{\mathrm{ref}} ; \omega\right)=\frac{1}{1+\gamma p(\omega)} \exp [-p(\omega) \Delta x]
$$

in a frequency domain, and

$$
\begin{aligned}
A\left(x, x_{\mathrm{ref}}=0 ; t\right)= & \gamma^{2} \exp \left(\gamma \Delta x+\gamma^{2} t\right)\left[1-\operatorname{erf}\left(\gamma \sqrt{t}+\frac{\Delta x}{2 \sqrt{t}}\right)\right] \\
& +\frac{1}{\sqrt{\pi t}} \gamma \exp \left(-\frac{\Delta x^{2}}{4 t}\right)
\end{aligned}
$$

in a time domain (see, for example, section 2.8 of Carslaw and Jaeger, 1986).

Once $\Delta T$ is determined for an arbitrary location, $\Delta E$ is determined as

$$
\Delta E(t)=a<\Delta T(t)>,
$$

where $\langle\Delta T>$ is the average of $\Delta T$ over the extensometer, and $a$ is the thermal-expansion coefficient of the extensometer. An approximation of $\langle\Delta T\rangle$ would be given by $\Delta T\left(x_{\mathrm{c}}, t\right)$ with a suitably chosen value of $x_{\mathrm{c}}$. Therefore, $\Delta E$ is given by

$$
\Delta E(t)=a \Delta T\left(x_{\mathrm{c}}, t\right)
$$

Parameters $a$ and $x s c$ should be determined empirically. If the reduction to Eq. (4) is valid, variations in temperature can be estimated; therefore, false variations in strain data can be estimated. The actual signal in the strain data is therefore obtained as the difference between the recorded and estimated values, $\Delta E$.

\section{Outline of sample data}

We test the validity of the procedure described in Section 2 by using temperature and strain data from a vault at Sukumo $\left(\mathrm{N} 32.951^{\circ}, \mathrm{E} 132.813^{\circ}\right.$; herein referred to as

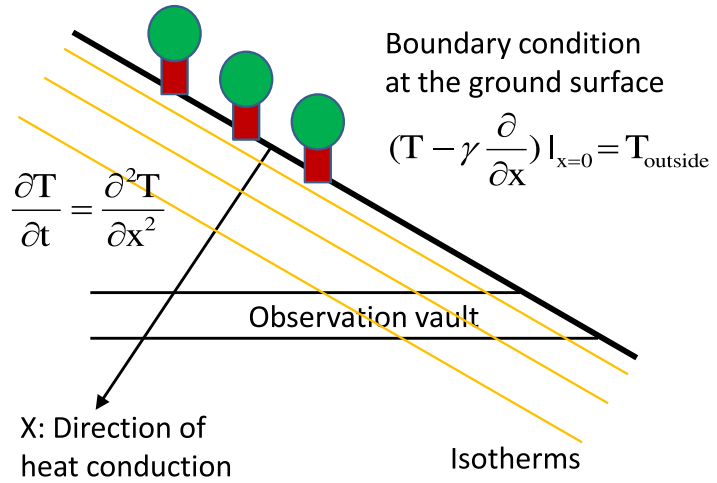

Figure 2: Schematic of heat conduction through the ground where a vault exists. $T$ is the temperature at a point in the ground, $T_{\text {ext }}$ is the atmospheric temperature, $t$ is time, $x$ is the location, and $\gamma$ is a constant representing heat conduction from the atmosphere to the ground. In this example, near the ground surface the heat conduction is not one-dimensional (i.e., isotherms are not planar), but at depth they are approximately one-dimensional (i.e., isotherms are planar).

SKM), near Bungo Channel in southwest Japan (Fig. 3). Ground strain has been observed using extensometers in the vault since 1987. The layout of the vault is shown in Fig. 4. Three extensometers (E1, E2, and E3), made from super-invar rods, are installed in the vault in three directions. The lengths of E1 and E2 are $10.50 \mathrm{~m}$, and the length of E3 is $5.25 \mathrm{~m}$.

Strain data at SKM are of interest for tectonic studies, in particular for slow slip events, for the following reasons. Beneath the Bungo Channel, long-term slow slip events (SSEs), with durations of about $1 \mathrm{yr}$, have been measured by GPS, starting in 1996 (e.g., Hirose et al., 1999; Ozawa et al., 2007), and resuming in 2002 (e.g., Ozawa et al., 2004, 2007) and 2010 (Hirose et al., 2010). Before the mid-1990s, GPS data were not available for investigating the SSEs in this area. Although analyses of leveling and sea level data suggest long-term SSEs also occurred around 1980, 1985, and 1991 (Kobayashi and Yamamoto, 2011), the magnitudes of individual events are uncertain. If the strain data at SKM are sufficiently accurate, they will help us quantify SSEs that took place before GPS was deployed.

Temporal variations in strain obtained with E1, E2, and E3 (referred to as E1, E2, and E3, respectively) are shown in Fig. 5. The original time series contains unnaturally large drifts in values, possibly due to some process of relaxation that occurs following installation of the instruments. To remove this drift, exponential 


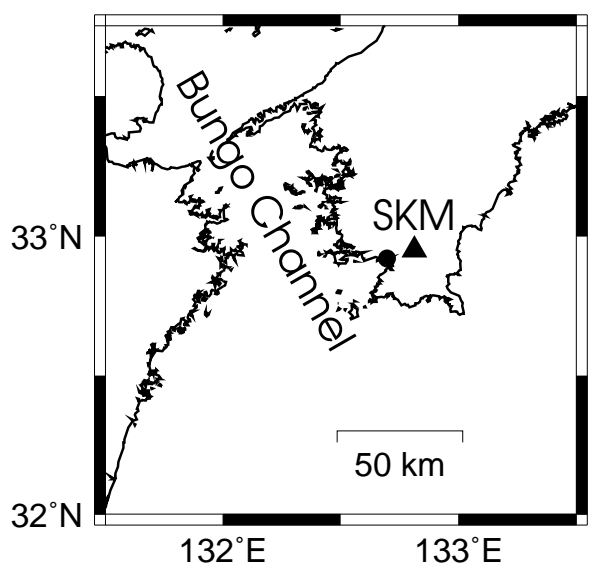

Figure 3: Locations of the Sukumo (SKM) site (triangle) and the nearest station (circle) of the Automated Meteorological Data Acquisition System (AMeDAS) of the Japan Meteorological Agency.

curves have been fitted to the original data, and the fitted curves are then removed from the original time series (Fig. 5b). In the numerical test, described in Section 4, we refer to the time series in Fig. 5b as "recorded values".

Temperature variations inside the vault are measured with thermometers installed at two points (T1 and T2, Fig. 4). These thermometers were installed in 1996. The temperature data of $\mathrm{T} 1$ and $\mathrm{T} 2$, denoted by $T_{1}$ and $T_{2}$ (Fig. 6), respectively, are used here for numerical testing of the procedure of estimating temperatures. The only temperature data available from before 1996 were measured from outside the vault. Atmospheric temperature data are available from the observatories of the Automated Meteorological Data Acquisition System (AMeDAS) operated by the Japan Meteorological Agency, and the nearest station for our purposes is at $\mathrm{N} 32.920^{\circ}$, E132.695 ${ }^{\circ}$ (Fig. 3). We use the temperature data from this station (denoted by $T_{\text {ext }}$ ) as reference temperatures for outside the vault.

\section{Estimating variations in temperature and strain using reference data}

In the situation of 1-D heat conduction, variations in temperature at a point $x$ can be estimated only when reference temperature data are available for a small $x_{\text {ref }}$. This condition is equivalent to the following statement: the phase of variations at the reference point should be preceded by the phase of variations at the target point.

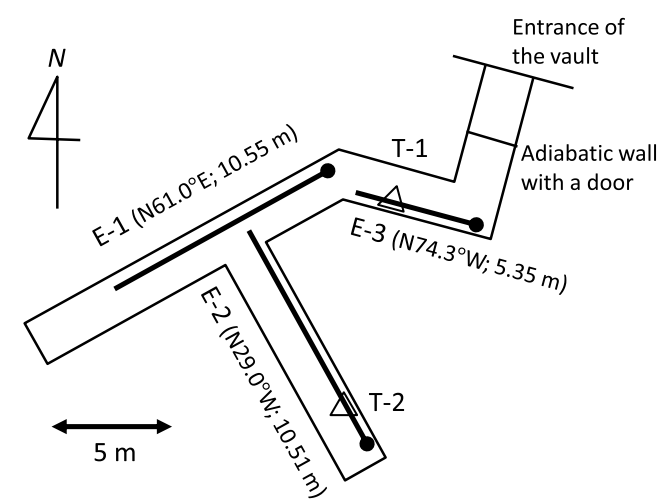

Figure 4: Plan view of the vault at SKM. Thick bars represent extensometers. The orientation and length of each extensometer are shown in parentheses. Open triangles represent the locations of thermometers.

Therefore, it is important to check the phase of each time series. Since annual components have relatively large amplitudes, we can check the phases of annual variations in temperature or strain data in order to consider which pair of time series can be estimated.

The phases and amplitudes of annual variations in strain and temperature data are summarized in Fig. 7. With regard to temperature, the phase of $\Delta T_{1}$ is preceded by the phases of $\Delta T_{2}$; hence, $\Delta T_{2}$ can possibly be estimated by $\Delta T_{1}$. We examine whether $\Delta T_{2}$ can be estimated by $\Delta T_{1}$ by checking whether the variations in temperature inside the vault can be estimated by reference data from inside the same vault. We also examine whether $\Delta T_{1}$ and $T_{2}$ can be estimated by $\Delta T_{\text {ext }}$ to check whether variations in temperature inside the vault can be estimated when the only available reference temperatures are from outside the vault. With regard to strain, we cannot anticipate estimating $\Delta E_{2}$ and $\Delta E_{3}$ by $\Delta T_{1}$ because the phases of $\Delta E_{2}$ and $\Delta E_{3}$ are preceded by the phases of reference temperatures inside the vault (i.e., $\Delta T_{1}$ and $\Delta T_{2}$ ). Therefore, we can only consider estimating false variations in $\Delta E_{1}$. We also examine how $\Delta E_{1}$ is estimated by variations in the reference temperatures outside the vault $\left(\Delta E_{\text {ext }}\right)$.

\subsection{Estimating variations in temperature using refer- ence data from inside the vault}

First, we try to estimate variations in temperature at a point $\left(\Delta T_{2}\right)$ by using reference temperatures at another point inside the vault $\left(\Delta T_{1}\right)$ in terms of Eqs (1) and (8). A parameter to be adjusted is the difference in location between $\mathrm{T} 1$ and $\mathrm{T} 2\left(\Delta x=x_{2}-x_{1}\right.$, where $x_{1}$ and $x_{2}$ are locations of $\mathrm{T} 1$ and $\mathrm{T} 2$, respectively) in Eq. (8). Eq. 
(a) Without data processing

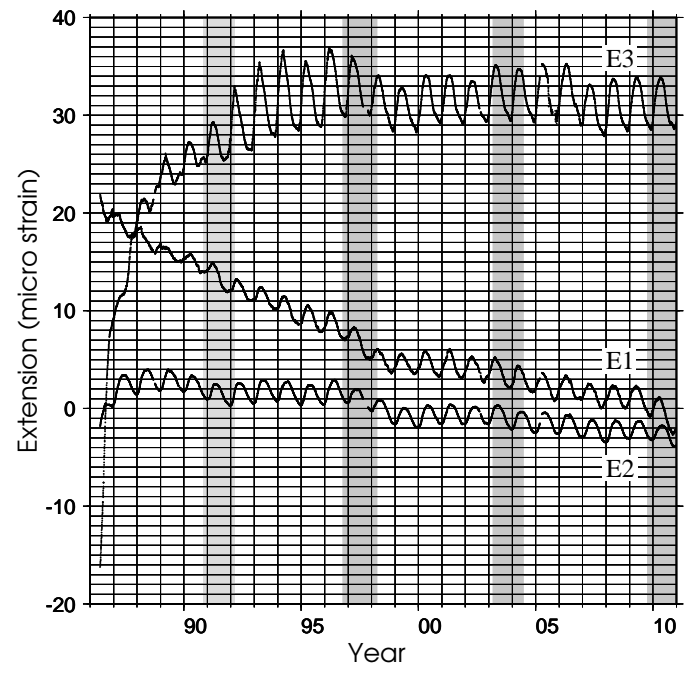

(b) After removing exponeitial trends

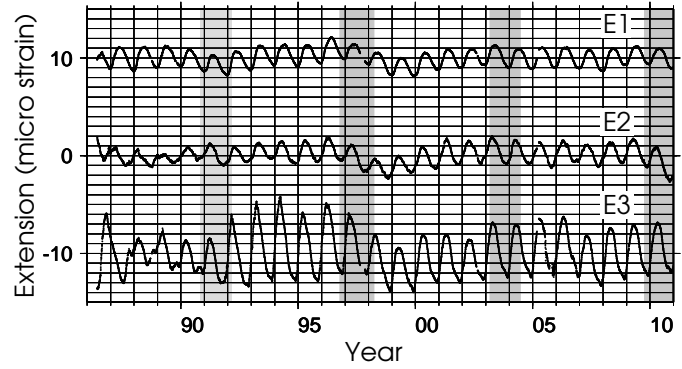

Figure 5: Variations in strain recorded by three extensometers at SKM. (a) Original time series. (b) Time series after exponential curves are removed from the original time series. Shadows represent periods of long-term slow slip events (SSE) beneath Bungo Channel. SSEs around 1997, 2003, and 2010 have been directly confirmed by GPS (e.g., Ozawa et al., 2007), while one event around 1991 is suggested by an analysis of sea level data (Kobayashi and Yamamoto, 2011).
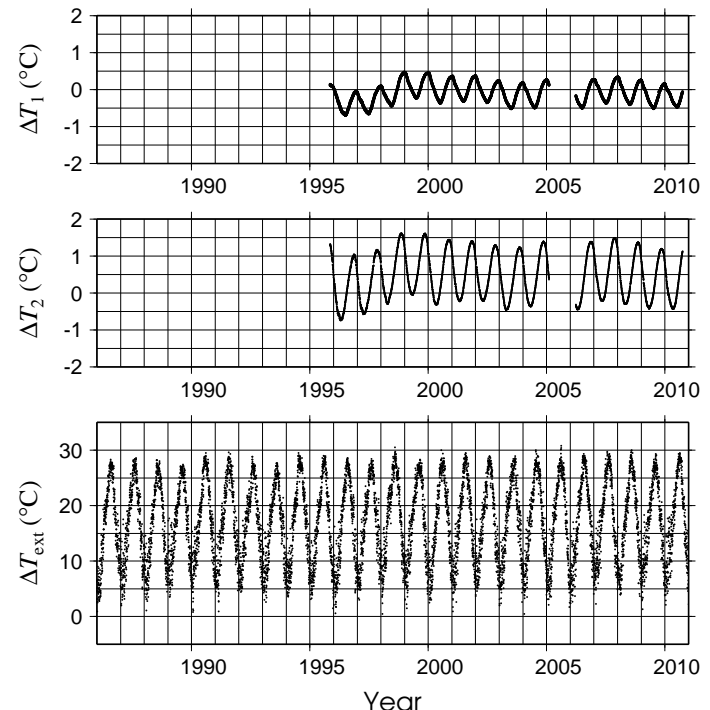

Figure 6: Variations in temperature at T1 and T2 in Fig. $4\left(\Delta T_{1}\right.$ and $\Delta T_{2}$, respectively) and at the nearest AMeDAS station $\left(\Delta T_{\text {ext }}\right)$.

(1) involves an integral over an infinite range, but we approximate it by a finite range of 1 year.

Figure 8 compares recorded and estimated variations in temperature at $\mathrm{T} 1$, together with their differences. The figure shows a good agreement between these two time series. Annual variations are still seen in the residual (lower panel), but they are only up to $0.1^{\circ} \mathrm{C}$. Moreover, no secular variation in the residual is seen. Since the coefficient of expansion of the super-invar rod is about $1-2 \times 10^{-60} \mathrm{C}$ (e.g., Furuzawa et al., 1993), a fluctuation in temperature of $0.1{ }^{\circ} \mathrm{Cis}$ equivalent to a $1-2 \times 10^{-7}$ fluctuation in strain in the strain data. Therefore, we can anticipate that false variations in strain data that result from variations in temperature can be removed with an accuracy better than $10^{-7}$ using reference temperature data from inside the vault.

\subsection{Estimating variations in temperature using refer- ence data from outside the vault}

Next, we try to estimate temperature variations inside the vault $\left(\Delta T_{1}\right.$ and $\left.\Delta T_{2}\right)$ by using reference external (i.e., outside the vault) temperatures $\left(\Delta T_{\text {ext }}\right)$ in terms of Eqs (1) and (12). Adjustable parameters are the locations of the thermometers $\left(x_{1}\right.$ and $\left.x_{2}\right)$ and the propagation coefficient between air and ground $(\gamma)$. However, the difference $x_{1}-x_{2}$ is equal to $\Delta x$, which is determined above; hence, only one of $x_{1}$ and $x_{2}$ is set freely. Reference temperatures from outside the vault are available after 1984. The integration in Eq. (12) is done over 3 yrs. 


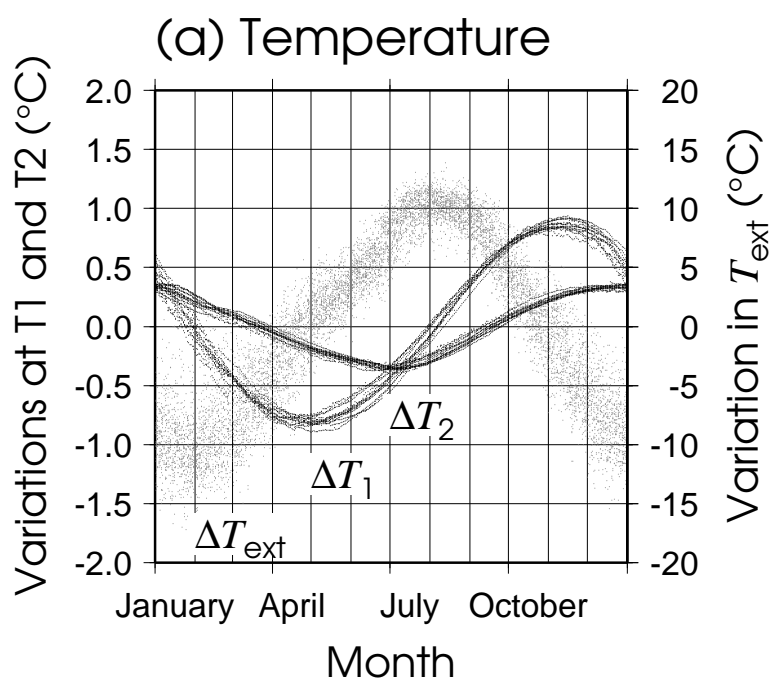

(b) Recorded strain

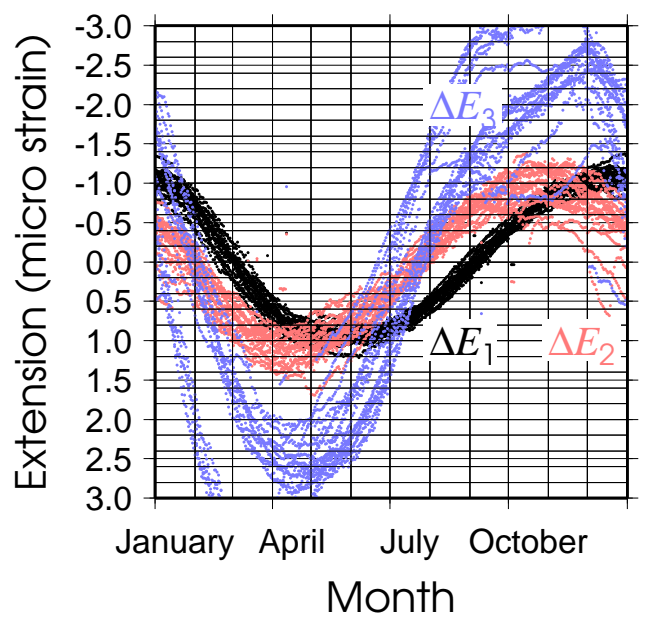

Figure 7: Stacked graphs showing annual (seasonal) variations in (a) temperature and (b) strain. (a) Curves in black indicate $\Delta T_{1}$ and $\Delta T_{2}$, and curves in gray indicate $\Delta T_{\text {ext }}$. (b) Curves in black, red, and blue indicate $\Delta E_{1}, \Delta E_{2}$, and $\Delta E_{3}$.
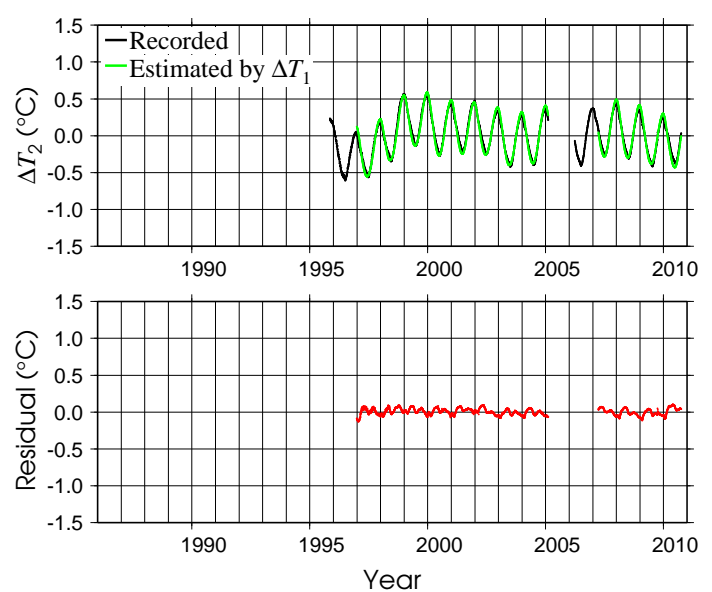

Figure 8: Recorded and estimated values of $\Delta T_{2}$ using reference temperature data from inside the vault $\left(\Delta T_{1}\right)$, together with their differences.

The parameter $x_{1}$ is determined in such a way that estimated variations of $\Delta T_{1}$ and $\Delta T_{2}$ mainly fit recorded variations of $\Delta T_{1}$ and $\Delta T_{2}$.

Figures 9 and 10 compare recorded and estimated variations in temperature at $\mathrm{T} 1$ and $\mathrm{T} 2$, respectively, together with their differences. For both $\Delta T_{1}$ and $\Delta T_{2}$, the annual variations seem to be successfully estimated so that the annual variations in residuals are reduced to $0.4-0.5^{\circ} \mathrm{Cin}$ peak-to-peak values. Since the recorded variations include annual variations in amplitude that are larger than $1.5^{\circ} \mathrm{Cin} \Delta T_{1}$ and $0.7^{\circ} \mathrm{Cin} \Delta T_{2}$, the estimated variations using reference data from outside the vault are considered to be reasonable. Yet, the accuracy is not as good as in the case of Fig. 8, for which the reference temperature data came from inside the vault. Concerning $\Delta T_{1}$, secular variations of up to 0.4 ${ }^{\circ} \mathrm{Cyr}^{-1}$ are largely reduced; even so, variations of approximately $0.05^{\circ} \mathrm{Cyr}^{-1}$ remain in the residual time series (e.g., during 2002-2004). Secular variations with approximately the same magnitude are also seen in the residual of $\Delta T_{2}$, and in this respect, the results differ from those obtained when temperature data from inside the vault are used.

\subsection{Estimating false variations in strain using refer- ence temperature data}

Finally, we try to estimate false variations in strain of $\mathrm{E} 1\left(\Delta E_{1}\right)$ by using variations in reference temperatures $\left(\Delta T_{1}\right.$ or $\left.\Delta T_{\text {ext }}\right)$ in terms of Eq. (14). The problems are in estimating the coefficient of expansion of the extensometer (a), and determining $\Delta T$ in Eq. (14). 

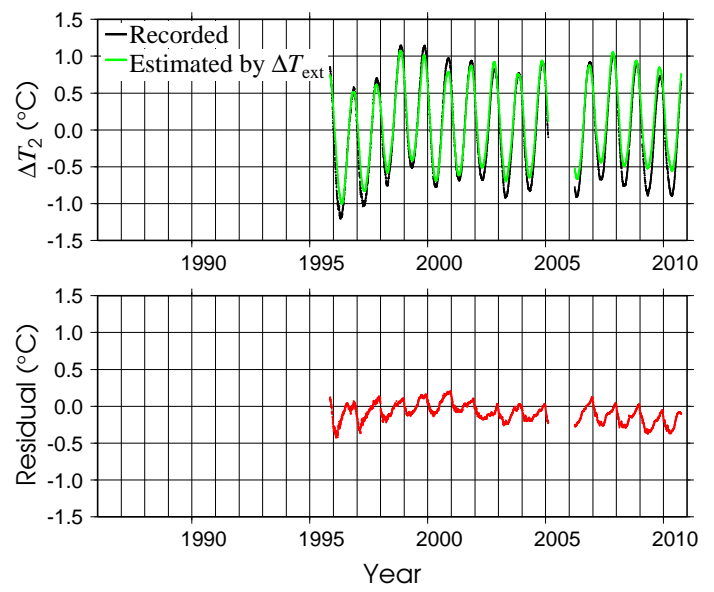

Figure 9: Recorded and estimated values of $\Delta T_{2}$ using reference temperature data from outside the vault $\left(\Delta T_{\text {ext }}\right)$, together with their differences.
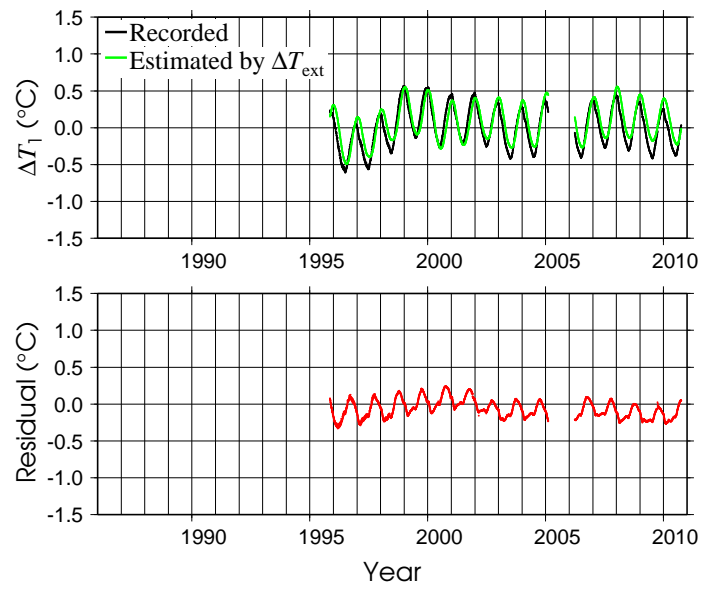

Figure 10: Recorded and estimated values of $\Delta T_{1}$ using reference temperature data from outside the vault $\left(\Delta T_{\text {ext }}\right)$, together with their differences.
When reference temperature variations are available from inside the vault $\left(\Delta T_{1}\right)$, the variation in temperature at E1 is estimated by Eqs (8) and (14). There are two parameters to be adjusted: $x_{\mathrm{c}}$ and $a$ in Eq. (14). Another parameter, $\Delta x$ in Eq. (8), is given by $\Delta x=x_{\mathrm{c}}-x_{1}$. These are determined in such a way that short-term (i year) variations in strain are reduced. A grid search is done for the fitting, and considering experimental results (e.g., Furuzawa et al., 1993), the coefficient of expansion $a$ is determined to be in the range $1<a<2$.

Figure 11 shows variations in recorded strain and false variations in strain estimated by using reference temperature variations from inside the vault, together with their differences. We see that annual variations in strain data are largely removed in the corrected time series. If Eq. (4) is valid, the optimum choices of parameters $\Delta x$ and $a$ to estimate annual variations are also the optimum choices for estimating secular variations. Therefore, we can anticipate that any secular variations that result from temperature variations are mostly removed, and the residuals reflect tectonic signals.

The results are consistent with other data. For the recorded variations in strain, changes in strain rates during the period 1996 to 1998 were approximately $2.0-3.0 \times 10^{-6}$. On the other hand, GPS data from stations near the SKM site indicate changes in strain during the same period of up to $1.0 \times 10^{-6}$ (F. Ohya, pers. comm.), which is inconsistent with the data from the extensometer (shown in Fig. 5). However, in the corrected time series, the changes in strain rate during the same period are up to $1.0 \times 10^{-6}$, which is considerably smaller than in the GPS data. This disappearance of the inconsistency between GPS and extensometer data suggests that even severely distorted strain data can be sometimes adequately corrected with reference temperature data from inside the vault.

When reference temperature variations are available only from outside the vault $\left(\Delta T_{\text {ext }}\right)$, the variations in temperature at E1 are estimated with Eqs (12) and (14). The locations of E1 ( $x$ in Eq. (12), and equivalently, $x_{\mathrm{c}}$ in Eq. (14)) and the coefficient of expansion ( $a$ in Eq. (14)) should be given, and these have already been determined in examples given above.

Figure 12 shows variations in recorded strain, and false variations in strain, estimated by reference temperature variations from outside the vault, together with their differences. Our interests are focused on detecting and quantifying the long-term slow slip event that may have occurred around 1990. In the residual time series, an offset of about $0.5-1.0^{-6}$ occurs between the trends for 1986-1989 and 1992-1995. However, estimations of inside temperatures using outside temperatures 

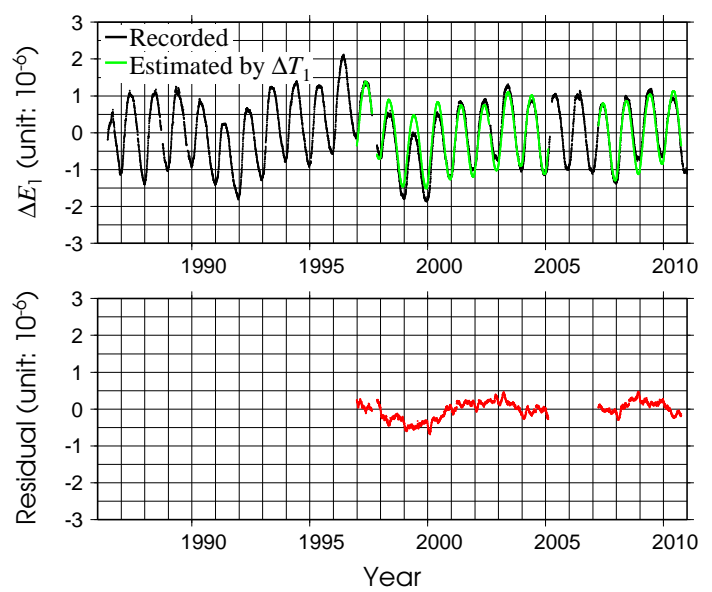

Figure 11: Recorded and estimated values of $\Delta E_{1}$ using reference temperature data from inside the vault $\left(\Delta T_{1}\right)$, together with their differences.

are subjected to significant errors, as seen in Figs. 9 and 10. Given that the estimated error in annual temperature variations is $0.1^{\circ} \mathrm{Cyr}^{-1}$, and that the coefficient of expansion is about $2.0 \times 10^{-6 \circ} \mathrm{C}^{-1}$, the strain data include errors of up to $0.6 \times 10^{-6}$, even after applying the corrections. Therefore, the offset in the trend around 1990 is not significantly larger than the errors anticipated from data corrections. What we can say is that the long-term slow slip event (SSE) around 1990 was not much larger than other SSEs.

\section{Discussion}

We have examined how temporal variations in temperature at a point inside a vault can be estimated by using reference variations in temperature at another point within the vault. Numerical testing shows that with regard to points $\mathrm{T} 1$ and $\mathrm{T} 2$ in the SKM vault, the variations in temperature at T2 $\left(\Delta T_{2}\right)$ are successfully estimated by the reference variations in temperature at $\mathrm{T} 1$ $\left(\Delta T_{1}\right)$, regardless of the major assumption and simplification of 1-D approximation (see Section 2 and Eq. (4) above). If the same procedure always works well, variations in temperature inside the vault can always be estimated with an accuracy of $0.1^{\circ} \mathrm{C}$, when the thermometer for reference is set at a suitable location. Moreover, it is possible to correct strain data with an accuracy of $10^{-7}$ when the thermal coefficients of expansion of the extensometers are $10^{-6 \circ} \mathrm{C}^{-1}$. This result indicates that we can possibly extract unrecognized events of crustal deformation on the order of $10^{-7}$, even when the extensometer is set in shallow vaults, if the reference temper-
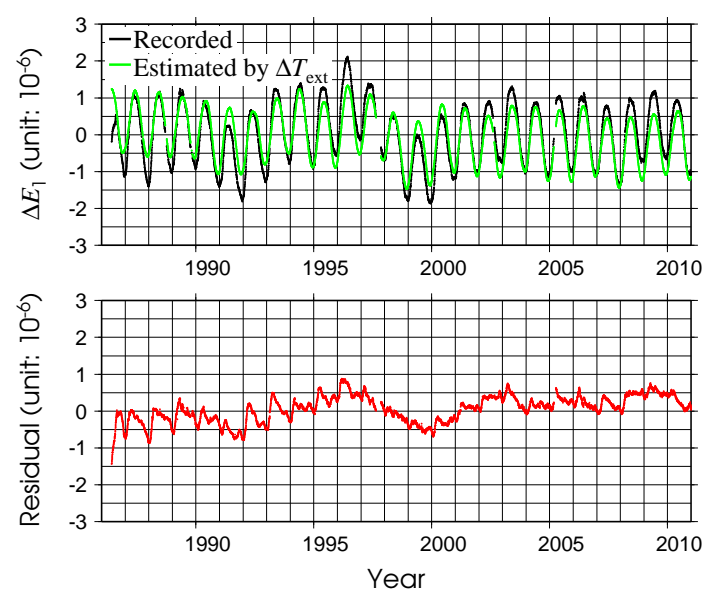

Figure 12: Recorded and estimated values of $\Delta E_{1}$ using reference temperature data from outside the vault $\left(\Delta T_{\text {ext }}\right)$, together with their differences.

ature is available inside the vault and the conditions of a layer of rock or soil around the vault are such that the thermo-elastic effect is relatively small.

However, as the vault at ISA was not equipped with thermometers in the pre-GPS era, extensometers at ISA cannot contribute to studies of crustal deformation unless their data are more precise than GPS data. When GPS stations are installed at intervals of $20-30 \mathrm{~km}$, as is the case in Japan, the detection limit of strain using GPS is on the order of $10^{-7}$. Given that the precision of strain data at ISA is up to $10^{-7}$ even after correction for false variations, there is no advantage in using vaulthoused extensometers over GPS. It is generally considered that strain data (measured by both borehole strainmeter and by extensometer) are more precise than GPS for short periods ( $;$ days) but less precise for longer time scales. In spite of the method proposed here to correct for thermal distortion, the problem of the low precision of long-term strain data remains to be unsolved.

The data also imply that heat conduction in some regions of the vault is unlikely to be described by a 1-D approximation, as indicated, for example, by the phases of T1 and E2. Considering the geometry of the vault (Fig. 4), T1 is closer to the entrance of the vault than E2; therefore, the phase of T1 should be preceded by the phase of E2 if heat conduction is one-dimensional. Nevertheless, this is not the case (Fig. 5a and b), possibly indicating that the direction of heat conduction is not simply from the surface to deep points at the locations of E2. The actual process of heat conduction around the vault is uncertain because there was only one pair of thermometers inside the vault. We need, therefore, 
to set multiple thermometers inside the vault, otherwise we cannot accurately evaluate the precision of the data correction, even if the corrections seem to work well. Since we lack such additional temperature data, we cannot discuss any further the precision of the corrections.

We have also attempted to estimate variations in temperature using reference variations in temperature from outside the vault. Estimated variations in temperature are, however, revealed to be relatively inaccurate, and the reasons for this are several. The most plausible reason is the unsuitable assumption about the boundary condition. Although heat generation due to the absorption of solar radiation has been totally ignored in our formulations, it should be included in the boundary condition. Another reason may be found in the temporal variations in the thermal properties of the ground. Temporal changes in the thermal diffusivity are indicated in Fig. 12. The annual variations are precisely corrected for the recent (i.e., after 2000) strain data, but imprecisely corrected for the old data (before the early 1990s), and this possibly indicates differences in the thermal diffusivities of the ground for these two periods. These temporal changes in thermal diffusivity may possibly be explained by changes in surface vegetation and water capacity near the surface, but they cannot be precisely quantified.

From the discussion above, we can see clearly that corrections of data in terms of temperature variations are far more difficult than anticipated. Among the many factors involved in the distortions of data derived from vault-housed extensometers, it had been thought that variations in temperature could be easily removed, based on the understanding that heat conduction is fundamentally a linear process, while other factors, including precipitation, are non-linear. However, our study has demonstrated that this is not the case, at least for some vaults. Indeed, variations in the temperature of an extensometer are revealed to be unpredictable unless a reference thermometer is installed at a suitable location. To estimate variations in temperature at arbitrary points inside the vault, a sufficient number of thermometers need to be installed. Unfortunately, few vaults are equipped with multiple thermometers.

A lesson learnt from our work is that efforts to search for tectonic signals in the strain data from vault-housed extensometers are likely to end in failure. This does not mean that vault-housed extensometers are of no use; indeed, they may provide essential information on phenomena whose time scales are rather short (i.e., i days). Because the short-term temporal variations in temperature within a vault are rather minor, short-term geophysical phenomena, including strain steps due to earthquakes and volcanic eruptions, can be successfully recorded without distortions from variations in temperature. However, if one's purpose is to study tectonic events with annual or longer time scales, we cannot recommend using shallow vaults for strain observations, because on those time scales the variations in temperature are significant.

\section{Conclusions}

Strain data obtained by vault-housed extensometers are influenced by two types of thermal noise: actual variations in strain of the crust due to the thermo-elastic effect, and false variations in strain due to the thermal expansion of the extensometer. False variations may be severe in the case of extensometers housed in shallow vaults. To correct for false variations in strain, we need to accurately estimate the variations in temperature at the location of the extensometer.

If temperature data from within the vault are available for reference, the variations in temperature in deeper parts of the vault may be predicted with an accuracy of $0.1^{\circ} \mathrm{C}$. In such a case, the strain data derived from the extensometer can be effectively corrected, even if the extensometer has been subjected to relatively large temperature variations of up to $1^{\circ} \mathrm{C}$. However, the precision achieved by this correction is insufficient to enable the use of strain data to uniquely constrain crustal deformation when dense GPS data are available. Given that the coefficient of expansion of an extensometer is about $1.0-2.0 \times 10^{-6 \circ} \mathrm{C}^{-1}$, fluctuations in temperature of $0.1^{\circ}$ Care equivalent to false variations in strain of $0.1-0.2 \times 10^{-6}$. An accuracy of $0.1 \times 10^{-6}$ is only comparable to the resolution of GPS data when GPS stations are installed at intervals of $20-30 \mathrm{~km}$, and in such a case there is no advantage in using vault-housed extensometers over GPS.

If the only available temperature data are obtained from outside the vault, estimated variations in temperature within the vault are insufficiently accurate to remove false variations in strain due to the variations in temperature. The available data indicate a secular variation in thermal diffusivity around vaults, and this creates further problems in estimating the variations in temperature.

In summary, we have found that strain data obtained with vault-housed extensometers are not easily corrected if the extensometers are influenced by variations in temperature. I conclude, therefore, that studies of long-term tectonic phenomena have little to gain by obtaining extensometer data in vaults that are subjected to variations in temperature. 


\section{Acknowledgements}

Our observations at SKM were conducted on private land with the kind permission of the landowner, and this work continues with staff from the DPRI, Kyoto University. AMeDAS data, provided by the Japan Meteorological Agency (JMA), were used after being downloaded from the JMA Web site. The software Generic Mapping Tools (Wessel and Smith, 1998) was used to prepare some of the illustrations. The author thanks two anonymous reviewers for providing thoughtful comments that considerably improved an early version of the manuscript.

This print is reproduced by the author to distribute the copyright material. A style file (elsarticle.cls) provided by the publisher (Elsevier B.V.) is used for typesetting. Minor differences, including layout and size of figures, may occur between this print and the formal version (Tectonophysics, volume 599, pages 89-96, 25 June 2013).

\section{References}

Ben-Zion, Y., Leary, P., 1986. Thermoelastic strain in a half-space covered by unconsolidated material. Bulletin of the Seismological Society of America 76(5), 1447-1460.

Berger, J., 1975. A note on thermoelastic strains and tilts. Journal of Geophysical Research 80, 274-277.

Carslaw, H.S., Jaeger, J.C., 1986. Conduction of Heat in Solids, second ed. Oxford University Press, Oxford.

Furuzawa, T., Ohya, F., Teraishi, M., Sonoda, Y., 1993. Test observation of crustal strains by two extensometers with super-invar rods of different coefficients of expansion. Journal of the Geodetic Society of Japan 39(4), 363-376.

Hagiwara, T., Rikitake, T., 1967. Japanese program on earthquake prediction. Science 157, 761-768.

Hashimoto, M., 2007. Intrinsic difficulties in the strategy of crustal deformation research based on continuous observation in vaults for earthquake prediction. Journal of the Geodetic Society of Japan 53(2), 183-195.

Harrison, J.C., Herbst, K., 1977. Thermoelastic strains and tilts revisited. Geophysical Research Letters 4, 535-537.

Hirose, H., Asano, Y., Obara, K., Kimura, T., Matsuzawa, T., Tanaka, S., Maeda, T., 2010. Slow earthquakes linked along dip in the Nankai subduction zone. Science 330, 1502.

Hirose, H., Hirahara, K., Kimata, F., Fujii, N., Miyazaki, S., 1999. A slow thrust slip event following the two 1996 Hyuganada earthquakes beneath the Bungo Channel, southwest Japan. Geophysical Research Letters 26, 3237-3240.

Ishihara, K., 1990. Pressure sources and induced ground deformation associated with explosive eruptions at an andesitic volcano: Sakurajima volcano, Japan, in: Ryan, M. (Ed.), Magma Transport and Storage. Wiley, New York, pp. 335-356.

Kasahara, M., Shichi, R., Okada, Y., 1983. On the cause of longperiod crustal movement. Tectonophysics 97, 327-336.

Kobayashi, A., Yamamoto, T., 2011. Repetitive long-term slow slip events beneath the Bungo Channel, southwestern Japan, identified from leveling and sea level data from 1979 to 2008. Journal of Geophysical Research 116, B04406.
Ozawa, S., Hatanaka, Y., Kaidzu, M., Murakami, M., Imakiire, T., Ishigaki, Y., 2004. Aseismic slip and low-frequency earthquakes in the Bungo channel, southwestern Japan. Geophysical Research Letters 31, L07609.

Ozawa, S., Suito, H., Imakiire, T., Murakami, M., 2007. Spatiotemporal evolution of aseismic interplate slip between 1996 and 1998 and between 2002 and 2004, in Bungo channel, southwest Japan. Journal of Geophysical Research 112, B05409.

Park, J., Amoruso, A., Crescentini, L., Boschi, E., 2008. Long-period toroidal Earth free oscillations from the great Sumatra-Andaman earthquake observed by paired laser extensometers in Gran Sasso, Italy. Geophysical Journal International 173, 887-905.

Teraishi, M., Onoue, K., Ohya, F., Nakamura, K., Takeuchi, F., Morii, W., Hoso, Y., Sonoda, Y., Wada, Y., 2009. Seasonal variations in the continuous observations of the crustal movement. Annuals of Disaster Prevention Research Institute, Kyoto University, 52B, 285-291. (In Japanese with English abstract and captions)

Wessel, P., Smith, W.H., 1998. Improved version of the Generic Mapping Tools released. EOS Transactions, AGU, 79, 579.

Yamaguchi, T., Kasahara, M., Takahashi, H., Okayama, M., Takada, M., Ichiyanagi, M., 2010. Development of Crustal Deformation Database System. Journal of the Geodetic Society of Japan 56, 4757.

Yamazaki, K., Teraishi, M., Ishihara, K., Komatsu, S., Kato, K., 2012. Subtle changes in strain prior to sub-Plinian eruptions recorded by vault-housed extensometers during the 2011 activity at Shinmoedake, Kirishima volcano, Japan. Earth, Planets and Space (under review).

Yamazaki, K., Teraishi, M., Komatsu, S., Sonoda, Y., Kano, Y., 2011. On the possibility of the 2011 Tohoku-oki earthquake reactivating Shinmoe-dake volcano, southwest Japan: insights from strain data measured in vaults. Natural Hazards and Earth System Sciences $11,2655-2661$. 